\title{
CURVATURE ESTIMATES FOR MINIMAL SUBMANIFOLDS OF HIGHER CODIMENSION
}

\author{
Y. L. XIN AND LING YANG
}

\begin{abstract}
We derive curvature estimates for minimal submanifolds in Euclidean space for arbitrary dimension and codimension via Gauss map. Thus, SchoenSimon-Yau's results and Ecker-Huisken's results are generalized to higher codimension. In this way we improve Hildebrandt-Jost-Widman's result for the Bernstein type theorem.
\end{abstract}

\section{INTRODUCTION}

Let $f$ be a smooth function on an open domain $\Omega \subset \mathbb{R}^{n}$. If $f$ satisfies the minimal surface equation, it defines a minimal hypersurface $M$ in $\mathbb{R}^{n+1}$.If $f$ is an entire solution to the equation, $f$ must be an affine linear function for $n \leq 7$ whose graph is a hyperplane. Those are the classical Bernstein theorem [2] and its higher dimensional generalizations which was finally proved by J. Simons [21] . Counterexamples to the theorems for $n \geq 8$ were given by Bombeiri-De Gorge-Guisti [1].

Heinze [11] considered the minimal graph defined over a disc $D_{R} \subset \mathbb{R}^{2}$ and gave curvature estimates. The classical Benstein theorem can be obtained by letting $R \rightarrow+\infty$ in his curvature estimates.

For general minimal surface in Euclidean space, so-called parametric case, the Beinstein type results are closely related to the value distribution of the Gauss image. The work of Osserman-Xavier-Fujimoto [18][25][8] has settled the question of the value of Gauss map for complete minimal surfaces in $\mathbb{R}^{3}$.

A minimal graph is area-minimizing, in particular, it is stable in the sense that its second variation of the volume is non-negative on any compact subset of $M$. Any stable minimal surface in $\mathbb{R}^{3}$ is a plane, due to Fisher- Colbrie- Schoen and do Carmo-Peng [7][5]. But, for higher dimensional stable hypersurfaces Cao-Shen-Zhu proved that they have only one end [3].

For stable minimal hypersurfaces, Schoen-Simon-Yau gave curvature estimates, which not only gave us a direct proof for Bernstein type theorems for $n \leq 5$ dimensional minimal graphs, but also gave us a new method to obtain curvature estimates.

1991 Mathematics Subject Classification. 49Q05, 53A07, 53A10.

The research was partially supported by NSFC. 
For any $n \geq 2$, there is a weak version of the Bernstein type theorem. It was J. Moser [16] who proved that the entire solution $f$ to the minimal surface equation is affine linear, provided $|\nabla f|$ is uniformly bounded. There is no dimension limitation. By a geometric approach Ecker-Huisken [10] obtained the curvature estimates, as a conclusion Moser's result has been improved for the controlled growth of $|\nabla f|$.

For area-minimizing hypersurfaces with vanishing first Betti number , Solomom [23] was able to give curvature estimates under the hypotheses of Gauss map.

Higher codimensional Bernstein problem becomes more complicated. There is a counterexample given by Lawson-Osserman [14]. On the other hand, HildebrandtJost-Widman [12] generalized Moser's result to higher codimension as follows.

Theorem 1.1. Let $z^{\alpha}=f^{\alpha}(x), \alpha=1, \cdots, m, x=\left(x^{1}, \cdots, x^{n}\right) \in \mathbb{R}^{n}$ be the $C^{2}$ solution to the system of minimal surface equations. Let there exist $\beta$, where

$$
\beta<\cos ^{-p}\left(\frac{\pi}{2 \sqrt{p K}}\right), \quad K=\left\{\begin{array}{ll}
1 & \text { if } p=1 \\
2 & \text { if } p \geq 2
\end{array} \quad, \quad p=\min (m, n)\right.
$$

such that for any $x \in \mathbb{R}^{n}$,

$$
\Delta_{f}(x)=\left\{\operatorname{det}\left(\delta_{i j}+f_{x^{i}}^{s}(x) f_{x^{j}}^{s}(x)\right)\right\}^{\frac{1}{2}} \leq \beta,
$$

then $f^{1}, \cdots, f^{m}$ are affine linear functions on $\mathbb{R}^{n}$, whose graph is affine $n$-plane in $\mathbb{R}^{m+n}$.

The geometric meaning of the condition in the above theorem is that the image under the Gauss map lies in a closed subset of an open geodesic ball of the radius $\frac{\sqrt{2}}{4} \pi$. Later in a joint work of the first author with J. Jost [13] we found larger geodesic convex set $B_{J X}\left(P_{0}\right)$, where $P_{0}$ denotes a fixed $n$-plane, and then improved the above theorem. Our bound of slope is 2 , big than $\cos ^{-p}\left(\frac{\pi}{2 \sqrt{p K}}\right)$. It should be noted that although $B_{J X}\left(P_{0}\right) \supset B_{\frac{\sqrt{2}}{4} \pi}\left(P_{0}\right)$, they have some common boundary points.

Recently, the first author and his collaborators [28][22] studied complete minimal submanifolds with flat normal bundle and positive $w$-function. In this special situation, the Schoen-Simon-Yau type curvature estimates and the Ecker-Huisken type curvature estimates can be carried out, then the corresponding Bernstein type theorems follow immediately.

In this paper we study a complete minimal submanifold $M$ in $\mathbb{R}^{m+n}$ with the codimension $m \geq 2$. We have a Bochner type formula for the squared norm of the second fundamental form $B$. As in the codimension one case, we need a Kato type inequality for $|\nabla B|^{2}$ in terms of $|\nabla| B||^{2}$. This is one of the crucial issue in the curvature estimates. We derive it for any codimension in $\S 2$.

Since the curved normal bundle, the curvature estimates would be more delicate. We can define Gauss map from $M$ to the Grassmannian manifold $\mathbf{G}_{n, m}$. From 
the counterexample of Lawson-Osserman, some additional conditions are needed to study higher codimensional Bernstein problem. The adequate conditions would confine the image of the Gauss map, as in the previous work of Osserman-XavierFujimoto in dimension 2 and in the work of Solomon in higher dimension. Now, in general dimension and codimension, we assume that the image under the Gauss map lies in an open geodesic ball of radius $\frac{\sqrt{2}}{4} \pi$ in $\mathbf{G}_{n, m}$ which is the largest convex geodesic ball in Grassmannian. We find two auxiliary functions $h_{1}$ and $h_{2}$ on $M$ via Gauss map. The precise definition and their properties can be found in $\S 3$.

(3.4) shows that $h_{1}$ can be viewed as a generalized support function. With the aid of the function $h_{1}$, we can derive a "strong stability inequality" which enables us to carry out the curvature estimates of Schoen-Simon-Yau type.

Using the function $h_{2}$ we can find subharmonic functions on $M$ and carry out the curvature estimates of Ecker-Huisken type in terms of $h_{2}$.

By those curvature estimates we can obtain the following Bernstein type theorems.

Theorem 1.2. Let $M$ be a complete minimal $n$-submanifold in $\mathbb{R}^{n+m}(n \leq 6)$. If the Gauss image of $M$ is contained in an open geodesic ball of $\mathbf{G}_{n, m}$ centered at $P_{0}$ and of radius $\frac{\sqrt{2}}{4} \pi$, then $M$ has to be an affine linear subspace.

Theorem 1.3. Let $M$ be a complete minimal n-submanifold in $\mathbb{R}^{n+m}$. If the Gauss image of $M$ is contained in an open geodesic ball of $\mathbf{G}_{n, m}$ centered at $P_{0}$ and of radius $\frac{\sqrt{2}}{4} \pi$, and $\left(\frac{\sqrt{2}}{4} \pi-\rho \circ \gamma\right)^{-1}$ has growth

$$
\left(\frac{\sqrt{2}}{4} \pi-\rho \circ \gamma\right)^{-1}=o(R)
$$

where $\rho$ denotes the distance on $\mathbf{G}_{n, m}$ from $P_{0}$ and $R$ is the Euclidean distance from any point in $M$. Then $M$ has to be an affine linear subspace.

In general, if there is neither dimension limitation as in Theorem 1.2 nor the growth assumption as in Theorem 1.3, we conclude that $M$ has only one end which is a consequence of the "strong stability inequality".

Our Theorem 1.2 and Theorem 1.3 are closely related to Theorem 1.1 mentioned above. Our results are higher codimensional generalizations of Schoen-Simon-Yau's results and Ecker-Huisken's results, and improve Hildebrandt-Jost-Widman's theorem.

It is worthy to note that our method is also suitable for codimension one case. We only need to modify the auxiliary functions $h_{1}$ and $h_{2}$ in order to recover the known results for minimal hypersurfaces. 


\section{A Kato-Type Formula}

Let $M \rightarrow \bar{M}$ be an isometric immersion with the second fundamental form $B$, which can be viewed as a cross-section of the vector bundle $\operatorname{Hom}\left(\odot^{2} T M, N M\right)$ over $M$, where $T M$ and $N M$ denote the tangent bundle and the normal bundle along $M$, respectively. A connection on $\operatorname{Hom}\left(\odot^{2} T M, N M\right)$ can be induced from those of $T M$ and $N M$ naturally.

For $\nu \in \Gamma(N M)$ the shape operator $A^{\nu}: T M \rightarrow T M$ satisfies

$$
\left\langle B_{X Y}, \nu\right\rangle=\left\langle A^{\nu}(X), Y\right\rangle .
$$

We define the mean curvature $H$ to be the trace of the second fundamental form. It is a normal vector field on $M$ in $\bar{M}$.

The second fundamental form, curvature tensor of the submanifold, curvature tensor of the normal bundle and that of the ambient manifold satisfy the Gauss equations, the Codazzi equations and the Ricci equations as follows.

$$
\begin{gathered}
\left\langle R_{X Y} Z, W\right\rangle=\left\langle\bar{R}_{X Y} Z, W\right\rangle-\left\langle B_{X W}, B_{Y Z}\right\rangle+\left\langle B_{X Z}, B_{Y W}\right\rangle, \\
\left(\nabla_{X} B\right)_{Y Z}-\left(\nabla_{Y} B\right)_{X Z}=-\left(\bar{R}_{X Y} Z\right)^{N}, \\
\left\langle R_{X Y} \mu, \nu\right\rangle=\left\langle\bar{R}_{X Y} \mu, \nu\right\rangle+\left\langle B_{X e_{i}}, \mu\right\rangle\left\langle B_{Y e_{i}}, \nu\right\rangle-\left\langle B_{X e_{i}}, \nu\right\rangle\left\langle B_{Y e_{i}}, \mu\right\rangle,
\end{gathered}
$$

where $\left\{e_{i}\right\}$ is a local orthonormal frame field of $M ; X, Y$ and $Z$ are tangent vector fields; $\mu, \nu$ are normal vector fields in $M$. Here and in the sequel we use the summation convention and agree the range of indices:

$$
1 \leq i, j, s, t \leq n ; \quad 1 \leq \alpha, \beta, \gamma \leq m ; \quad 1 \leq a, b, c \leq m+n .
$$

There is the trace-Laplace operator $\nabla^{2}$ acting on any cross-section of a Riemannian vector bundle $E$ over $M$.

Now, we consider a minimal submanifold $M$ of dimension $n$ in Euclidean $(m+n)$ space $\mathbb{R}^{m+n}$ with $m \geq 2$. We have (see [21])

$$
\nabla^{2} B=-\tilde{\mathcal{B}}-\underline{\mathcal{B}} .
$$

We recall the following notations:

$$
\tilde{\mathcal{B}} \stackrel{\text { def. }}{=} B \circ B^{t} \circ B
$$

where $B^{t}$ is the conjugate map of $B$;

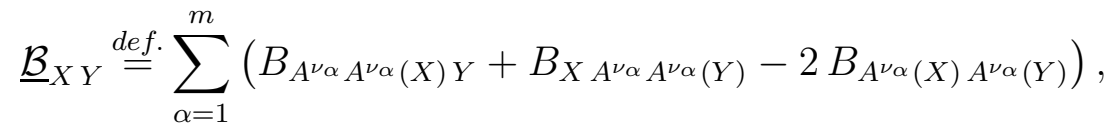

where $\nu_{\alpha}$ are basis vectors of normal space. It is obvious that $\underline{\mathcal{B}}_{X Y}$ is symmetric in $X$ and $Y$, which is a cross-section of the bundle $\operatorname{Hom}\left(\odot^{2} T M, N M\right)$. Simons also gave an estimate [21]

$$
\langle\tilde{\mathcal{B}}+\underline{\mathcal{B}}, B\rangle \leq\left(2-\frac{1}{m}\right)|B|^{4} .
$$


It is optimal for the codimension $m=1$.

In the case when $m \geq 2$, there is a refined estimate [15][6]

$$
\langle\tilde{\mathcal{B}}+\underline{\mathcal{B}}, B\rangle \leq \frac{3}{2}|B|^{4}
$$

Substituting it into (2.1) gives

$$
\left\langle\nabla^{2} B, B\right\rangle \geq-\frac{3}{2}|B|^{4}
$$

It follows that

$$
\Delta|B|^{2} \geq-3|B|^{4}+2|\nabla B|^{2}
$$

We need a Kato-type inequality in order to use the formula (2.2). Namely, we would estimate $|\nabla B|^{2}$ in terms of $|\nabla| B||^{2}$. Schoen-Simon-Yau [20] did such an estimate for codimension $m=1$. For any $m$ with flat normal bundle their technique is also applicable, see the previous paper of the first author [28]. The following lemma is a generalized version of their estimate for any codimension $m$.

\section{Lemma 2.1.}

$$
|\nabla B|^{2} \geq\left(1+\frac{2}{m n}\right)|\nabla| B||^{2}
$$

Proof. It is sufficient for us to prove the inequality at the points where $|B|^{2} \neq 0$. Choose a local orthonormal tangent frame field $\left\{e_{1}, \cdots, e_{n}\right\}$ and a local orthonormal normal frame field $\left\{\nu_{1}, \cdots, \nu_{m}\right\}$ of $M$ near the considered point $x$, such that

$$
\nabla_{e_{i}} e_{j}(x)=0, \nabla_{e_{i}} \nu_{\alpha}(x)=0
$$

Denote the shape operator $A^{\alpha}=A^{\nu_{\alpha}}$. Then obviously $|B|^{2}=\sum_{\alpha}\left|A^{\alpha}\right|^{2}$ and

$$
\begin{aligned}
\nabla|B|^{2} & =\sum_{\alpha} \nabla\left|A^{\alpha}\right|^{2} . \\
\left.\left.|\nabla| B\right|^{2}\right|^{2} & =\sum_{\alpha, \beta} \nabla\left|A^{\alpha}\right|^{2} \cdot \nabla\left|A^{\beta}\right|^{2} \leq \frac{1}{2} \sum_{\alpha, \beta}\left(\left.\left.|\nabla| A^{\alpha}\right|^{2}\right|^{2}+\left.\left.|\nabla| A^{\beta}\right|^{2}\right|^{2}\right) \\
& \leq\left.\left. m \sum_{\alpha}|\nabla| A^{\alpha}\right|^{2}\right|^{2} .
\end{aligned}
$$

Therefore

$$
|\nabla| B||^{2}=\frac{\left.\left.|\nabla| B\right|^{2}\right|^{2}}{4|B|^{2}} \leq \frac{\left.\left.m \sum_{\alpha}|\nabla| A^{\alpha}\right|^{2}\right|^{2}}{4 \sum_{\alpha}\left|A^{\alpha}\right|^{2}}
$$

Since $|B|^{2} \neq 0$, there exist $\gamma, k, l$ such that $h_{\gamma k l} \neq 0$, where $h_{\alpha i j}=\left\langle B_{e_{i} e_{j}}, \nu_{\alpha}\right\rangle$ for arbitrary $\alpha, i, j$; then

$$
\left(h_{1 k l}, \cdots, h_{m k l}\right) \in \mathbb{R}^{m}-\{0\}
$$


Obviously, there exists an $m \times m$ orthogonal matrix $U$ and $z=\left(z_{1}, \cdots, z_{m}\right) \in \mathbb{R}^{m}$, such that

$$
z_{\alpha} \neq 0 \quad \text { for every } 1 \leq \alpha \leq m
$$

and

$$
z_{\alpha}=U_{\alpha}^{\beta} h_{\beta k l} .
$$

Now we define $\tilde{\nu}_{\alpha}=U_{\alpha}^{\beta} \nu_{\beta}$, then $\left\{\tilde{\nu}_{1}, \cdots, \tilde{\nu}_{m}\right\}$ is also a local orthogonal normal frame field, satisfying (2.4); and moreover

$$
\tilde{h}_{\alpha k l}=\left\langle B_{e_{k} e_{l}}, \tilde{\nu}_{\alpha}\right\rangle=U_{\alpha}^{\beta} h_{\beta k l}=z_{\alpha} \neq 0 .
$$

Define the shape operator $\tilde{A}^{\alpha}$ corresponding to $\tilde{\nu}_{\alpha}$, then

$$
\left|\tilde{A}^{\alpha}\right|^{2}=\sum_{i, j} \tilde{h}_{\alpha i j}^{2} \geq \tilde{h}_{\alpha k l}^{2}>0 .
$$

Hence we can assume $\left|A^{\alpha}\right|^{2}>0$ for arbitrary $\alpha$ without loss of generality.

Let $1 \leq \gamma \leq m$ such that

$$
\frac{\left.\left.|\nabla| A^{\gamma}\right|^{2}\right|^{2}}{\left|A^{\gamma}\right|^{2}}=\max _{\alpha}\left\{\frac{\left.\left.|\nabla| A^{\alpha}\right|^{2}\right|^{2}}{\left|A^{\alpha}\right|^{2}}\right\}<+\infty,
$$

then from $(2.5)$,

$$
|\nabla| B||^{2} \leq \frac{\left.\left.m|\nabla| A^{\gamma}\right|^{2}\right|^{2}}{4\left|A^{\gamma}\right|^{2}}
$$

$\left|A^{\gamma}\right|^{2}$ and $\nabla\left|A^{\gamma}\right|^{2}$ is independent of the choice of $\left\{e_{1}, \cdots, e_{n}\right\}$, then without loss of generality we can assume $h_{\gamma i j}=0$ whenever $i \neq j$. Then

$$
\begin{aligned}
\nabla\left|A^{\gamma}\right|^{2} & =2 \sum_{k} \sum_{i, j} h_{\gamma i j} h_{\gamma i j k} e_{k}=2 \sum_{k} \sum_{i} h_{\gamma i i} h_{\gamma i i k} e_{k}, \\
\left.\left.|\nabla| A^{\gamma}\right|^{2}\right|^{2} & =4 \sum_{k}\left(\sum_{i} h_{\gamma i i} h_{\gamma i i k}\right)^{2} \leq 4 \sum_{k}\left(\sum_{i} h_{\gamma i i}^{2}\right)\left(\sum_{i} h_{\gamma i i k}^{2}\right) \\
& =4\left(\sum_{i} h_{\gamma i i}^{2}\right)\left(\sum_{i, k} h_{\gamma i i k}^{2}\right)=4\left|A^{\gamma}\right|^{2} \sum_{i, k} h_{\gamma i i k}^{2}
\end{aligned}
$$

and

$$
\begin{aligned}
|\nabla| B||^{2} & \leq \frac{\left.\left.m|\nabla| A^{\gamma}\right|^{2}\right|^{2}}{4\left|A^{\gamma}\right|^{2}} \leq m \sum_{i, k} h_{\gamma i i k}^{2} \\
& =m \sum_{i \neq k} h_{\gamma i i k}^{2}+m \sum_{i} h_{\gamma i i i}^{2} \\
& =m \sum_{i \neq k} h_{\gamma i k i}^{2}+m \sum_{i}\left(\sum_{j \neq i} h_{\gamma j j i}\right)^{2} \\
& \leq m \sum_{i \neq k} h_{\gamma i k i}^{2}+(n-1) m \sum_{i \neq j} h_{\gamma j j i}^{2}
\end{aligned}
$$




$$
=n m \sum_{i \neq k} h_{\gamma i k i}^{2},
$$

where we used the Codazzi equations and the vanishing mean curvature condition $H=0$. Please note that $h_{\alpha i j k}=\left\langle\left(\nabla_{e_{k}} B\right)_{e_{i}, e_{j}}, \nu_{\alpha}\right\rangle$ for arbitrary $\alpha, i, j, k$.

On the other hand, a direct calculation shows

$$
\begin{aligned}
\left.\left.|\nabla| B\right|^{2}\right|^{2} & =\left|2 \sum_{k} \sum_{\alpha, i, j} h_{\alpha i j} h_{\alpha i j k} e_{k}\right|^{2}=4 \sum_{\alpha, \beta, i, j, s, t, k} h_{\alpha i j} h_{\alpha i j k} h_{\beta s t} h_{\beta s t k}, \\
|\nabla B|^{2}-\left.|\nabla| B\right|^{2} & =|\nabla B|^{2}-\frac{\left.\left.|\nabla| B\right|^{2}\right|^{2}}{4|B|^{2}} \\
& =\sum_{\alpha, i, j, k} h_{\alpha i j k}^{2}-\frac{\sum_{\alpha, \beta, i, j, s, t, k} h_{\alpha i j} h_{\alpha i j k} h_{\beta s t} h_{\beta s t k}}{\sum_{\beta, s, t} h_{\beta s t}^{2}} \\
& =\frac{\sum_{\alpha, i, j, s, t, k}\left(h_{\alpha i j k} h_{\beta s t}-h_{\beta s t k} h_{\alpha i j}\right)^{2}}{2|B|^{2}} \\
& \geq \frac{\sum_{\beta, i \neq j, s, t, k} h_{\gamma i j k}^{2} h_{\beta s t}^{2}+\sum_{\alpha, s \neq t, i, j, k} h_{\gamma s t k}^{2} h_{\alpha i j}^{2}}{2|B|^{2}} \\
& =\sum_{i \neq j, k} h_{\gamma i j k}^{2} \geq \sum_{i \neq k}\left(h_{\gamma i k i}^{2}+h_{\gamma i k k}^{2}\right) \\
& =2 \sum_{i \neq k} h_{\gamma i k i}^{2} .
\end{aligned}
$$

In conjunction with (2.7), we finally arrive at (2.3).

Hence it follows from (2.2) and (2.3) that

$$
\Delta|B|^{2} \geq\left. 2\left(1+\frac{2}{m n}\right)|\nabla| B\right|^{2}-3|B|^{4} .
$$

\section{Auxiliary Functions via Gauss Maps}

Let $\mathbb{R}^{m+n}$ be an $(m+n)$-dimensional Euclidean space. All oriented $n$-subspaces constitute the Grassmannian manifold $\mathbf{G}_{n, m}$, which is an irreducible symmetric space of compact type. The canonical Riemannian metric on $\mathbf{G}_{n, m}$ can be expressed in the following way.

Let $\left\{e_{i}, e_{n+\alpha}\right\}$ be a local orthonormal frame field in $\mathbb{R}^{m+n}$. Let $\left\{\omega_{i}, \omega_{n+\alpha}\right\}$ be the dual frame field so that the Euclidean metric is

$$
g=\sum_{i} \omega_{i}^{2}+\sum_{\alpha} \omega_{n+\alpha}^{2}
$$


The Levi-Civita connection forms $\omega_{a b}$ of $\mathbb{R}^{m+n}$ are uniquely determined by the equations

$$
\begin{aligned}
& d \omega_{a}=\omega_{a b} \wedge \omega_{b}, \\
& \omega_{a b}+\omega_{b a}=0 .
\end{aligned}
$$

Let $P \in \mathbf{G}_{n, m}$ be any point which is spanned by $\left\{e_{1}, \cdots, e_{n}\right\}$, then the canonical Riemannian metric on $\mathbf{G}_{n, m}$ can be written as

$$
d s^{2}=\sum_{i, \alpha} \omega_{n+\alpha i}^{2} .
$$

The sectional curvature of the above canonical metric varies in the interval $[0,2]$ in the case of $\min \{n, m\} \geq 2$. By the standard Hessian comparison theorem we have

$$
\operatorname{Hess}(\rho) \geq \sqrt{2} \cot (\sqrt{2} \rho)(g-d \rho \otimes d \rho),
$$

where $\rho$ is the distance function from a fixed point in $\mathbf{G}_{n, m}$ and $g$ is the metric tensor on $\mathbf{G}_{n, m}$.

Let 0 be the origin of $\mathbb{R}^{m+n}$. Let $S O(m+n)$ be the Lie group consisting of all the orthonormal frames $\left(0 ; e_{i}, e_{n+\alpha}\right)$. Let $P=\left\{\left(x ; e_{1}, \ldots, e_{n}\right): x \in M, e_{i} \in T_{x} M\right\}$ be the principal bundle of orthonormal tangent frames over $M, Q=\left\{\left(x ; e_{n+1}, \ldots, e_{n+m}\right)\right.$ : $\left.x \in M, e_{n+\alpha} \in N_{x} M\right\}$ be the principal bundle of orthonormal normal frames over $M$, then $\bar{\pi}: P \oplus Q \rightarrow M$ is the projection with fiber $S O(m) \times S O(n), i: P \oplus Q \hookrightarrow$ $S O(m+n)$ is the natural inclusion.

The Gauss map $\gamma: M \rightarrow \mathbf{G}_{n, m}$ is defined by

$$
\gamma(x)=T_{x} M \in \mathbf{G}_{n, m}
$$

via the parallel translation in $\mathbb{R}^{m+n}$ for $\forall x \in M$. Thus, the following commutative diagram holds

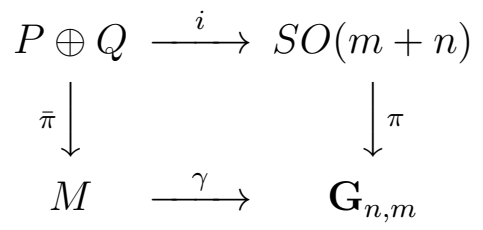

From the above diagram we know that the energy density of the Gauss map (see [26] Chap.3, §3.1)

$$
e(\gamma)=\frac{1}{2}\left\langle\gamma_{*} e_{i}, \gamma_{*} e_{i}\right\rangle=\frac{1}{2}|B|^{2}
$$

Ruh-Vilms proved that the mean curvature vector of $M$ is parallel if and only if its Gauss map is a harmonic map [19].

We consider smooth functions on an open geodesic ball $B_{\frac{\sqrt{2}}{4} \pi}\left(P_{0}\right) \subset \mathbf{G}_{n, m}$ of radius $\frac{\sqrt{2}}{4} \pi$ and centered at $P_{0}$. Those are useful for our curvature estimates later. 
Let

$$
u=\cos (\sqrt{2} \rho),
$$

where $\rho$ is the distance function from $P_{0}$ in $\mathbf{G}_{n, m}$. We have

$$
\begin{aligned}
& u^{\prime}=-\sqrt{2} \sin (\sqrt{2} \rho), \\
& u^{\prime \prime}=-2 \cos (\sqrt{2} \rho) .
\end{aligned}
$$

Then,

$$
\begin{aligned}
\operatorname{Hess}(u) & =u^{\prime} \operatorname{Hess}(\rho)+u^{\prime \prime} d \rho \otimes d \rho \\
& \leq-2 \cos (\sqrt{2} \rho)(g-d \rho \otimes d \rho)-2 \cos (\sqrt{2} \rho) d \rho \otimes d \rho=-2 u g
\end{aligned}
$$

The composition function $h_{1}=u \circ \gamma$ of $u$ with the Gauss map $\gamma$ defines a function on $M$. Using the composition formula, we have

$$
\begin{aligned}
\Delta h_{1} & =\operatorname{Hess}(u)\left(\gamma_{*} e_{i}, \gamma_{*} e_{i}\right)+d u(\tau(\gamma)) \\
& \leq-2|B|^{2} h_{1},
\end{aligned}
$$

where $\tau(\gamma)$ is the tension field of the Gauss map, which is zero, provided $M$ has parallel mean curvature by the Ruh-Vilms theorem mentioned above.

Let

$$
h=\sec ^{2}(\sqrt{2} \rho),
$$

where $\rho$ is the distance function from $P_{0}$ in $\mathbf{G}_{n, m}$. We have

$$
\begin{gathered}
h^{\prime}=2 \sqrt{2} \sec ^{2}(\sqrt{2} \rho) \tan (\sqrt{2} \rho), \\
h^{\prime \prime}=12 \sec ^{2}(\sqrt{2} \rho) \tan ^{2}(\sqrt{2} \rho)+4 \sec ^{2}(\sqrt{2} \rho) .
\end{gathered}
$$

Hence,

$$
\begin{aligned}
\operatorname{Hess}(h)= & h^{\prime} \operatorname{Hess}(\rho)+h^{\prime \prime} d \rho \otimes d \rho \\
\geq & 4 \sec ^{2}(\sqrt{2} \rho)(g-d \rho \otimes d \rho) \\
& \quad+\left(12 \sec ^{2}(\sqrt{2} \rho) \tan ^{2}(\sqrt{2} \rho)+4 \sec ^{2}(\sqrt{2} \rho)\right) d \rho \otimes d \rho \\
= & 4 h g+\frac{3}{2} h^{-1} d h \otimes d h
\end{aligned}
$$

The composition function $h_{2}=h \circ \gamma$ of $h$ with the Gauss map $\gamma$ defines a function on $M$. Using the composition formula, we have

$$
\begin{aligned}
\Delta h_{2} & =\operatorname{Hess}(h)\left(\gamma_{*} e_{i}, \gamma_{*} e_{i}\right)+d h(\tau(\gamma)) \\
& \geq 4 h_{2}|B|^{2}+\frac{3}{2} h_{2}^{-1}\left|\nabla h_{2}\right|^{2},
\end{aligned}
$$

where $\tau(\gamma)$ is the tension field of the Gauss map, which is zero in our consideration.

With the aid of $h_{1}$, we immediately have the following lemma. 
Lemma 3.1. Let $M$ be an $n$-dimensional minimal submanifold of $\mathbb{R}^{n+m}$ ( $M$ needs not be complete), if the Gauss image of $M$ is contained in an open geodesic ball of radius $\frac{\sqrt{2}}{4} \pi$ in $\mathbf{G}_{n, m}$, then we have

$$
\int_{M}|\nabla \phi|^{2} * 1 \geq 2 \int_{M}|B|^{2} \phi^{2} * 1
$$

for any function $\phi$ with compact support $D \subset M$.

Proof. Let

$$
L \phi=-\Delta \phi-2|B|^{2} \phi .
$$

Its first eigenvalue with the Dirichlet boundary condition in $D$ is $\lambda_{1}$ and the corresponding eigenfunction is $v$. Without loss of generality, we assume that $v$ achieves the positive maximum. Consider a $C^{2}$ function

$$
f=\frac{v}{h_{1}} \text {. }
$$

Since $\left.f\right|_{\partial D}=0$, it achieves the positive maximum at a point $x \in D$. Therefore, at $x$

$$
\nabla f=0, \quad \Delta f \leq 0 .
$$

It follows that

$$
\begin{aligned}
\Delta v & =\Delta\left(f h_{1}\right)=\Delta f \cdot h_{1}+f \Delta h_{1}+2 \nabla f \cdot \nabla h_{1} \\
& \leq f \Delta h_{1}=\frac{v \Delta h_{1}}{h_{1}} .
\end{aligned}
$$

Namely, at $x$,

$$
\begin{gathered}
\frac{\Delta v}{v} \leq \frac{\Delta h_{1}}{h_{1}} . \\
\frac{\Delta v+2|B|^{2} v}{v} \leq \frac{\Delta h_{1}+2|B|^{2} h_{1}}{h_{1}} \leq 0 .
\end{gathered}
$$

On the other hand,

$$
\frac{\Delta v+2|B|^{2} v}{v}=-\lambda_{1} .
$$

(3.7) and (3.8) implies $\lambda_{1} \geq 0$. Hence we have

$$
0 \leq \lambda_{1}=\inf \frac{\int_{D} \phi L \phi * 1}{\int_{D} \phi^{2} * 1} \leq \frac{\int_{D} \phi L \phi * 1}{\int_{D} \phi^{2} * 1},
$$

which shows (3.6) holds true.

Remark 3.1. For a stable minimal hypersurface there is the stability inequality, which is one of main ingredient for Schoen-Simon-Yau's curvature esimates for stable minimal hypersurfaces. For minimal submanifolds with the Gauss image restriction we have stronger inequality as shown in (3.6) 


\section{Curvature estimates of Schoen-Simon-Yau type}

Replacing $\phi$ by $|B|^{1+q} \phi$ in (3.6) gives

$$
\begin{aligned}
\int_{M}|B|^{4+2 q} \phi^{2} * 1 & \leq \frac{1}{2} \int_{M}\left|\nabla\left(|B|^{1+q} \phi\right)\right|^{2} * 1 \\
= & \frac{1}{2}(1+q)^{2} \int_{M}|B|^{2 q}|\nabla| B||^{2} \phi^{2} * 1+\frac{1}{2} \int_{M}|B|^{2+2 q}|\nabla \phi|^{2} * 1 \\
& +(1+q) \int_{M}|B|^{1+2 q} \nabla|B| \cdot \phi \nabla \phi * 1 .
\end{aligned}
$$

From (2.9) we can derive

$$
\frac{2}{m n}|\nabla| B||^{2} \leq|B| \Delta|B|+\frac{3}{2}|B|^{4} .
$$

Multiplying $|B|^{2 q} \phi^{2}$ with both sides of (4.2) and integrating by parts, we have

$$
\begin{aligned}
& \frac{2}{m n} \int_{M}|B|^{2 q}|\nabla| B||^{2} \phi^{2} * 1 \leq \int_{M}|B|^{1+2 q} \Delta|B| \phi^{2} * 1+\frac{3}{2} \int_{M}|B|^{4+2 q} \phi^{2} * 1 \\
&=-\int_{M} \nabla|B| \cdot \nabla\left(|B|^{1+2 q} \phi^{2}\right) * 1+\frac{3}{2} \int_{M}|B|^{4+2 q} \phi^{2} * 1 \\
&=-(1+2 q) \int_{M}|B|^{2 q}|\nabla| B||^{2} \phi^{2} * 1 \\
&-2 \int_{M}|B|^{1+2 q} \nabla|B| \cdot \phi \nabla \phi * 1+\frac{3}{2} \int_{M}|B|^{4+2 q} \phi^{2} * 1 .
\end{aligned}
$$

By multiplying $\frac{3}{2}$ with both sides of (4.1) and then adding up both sides of it and (4.3), we have

$$
\begin{aligned}
& \left(\frac{2}{m n}+1+2 q-\frac{3}{4}(1+q)^{2}\right) \int_{M}|B|^{2 q}|\nabla| B||^{2} \phi^{2} * 1 \\
& \leq \frac{3}{4} \int_{M}|B|^{2+2 q}|\nabla \phi|^{2} * 1+\left(\frac{3}{2}(1+q)-2\right) \int_{M}|B|^{1+2 q} \nabla|B| \cdot \phi \nabla \phi * 1 .
\end{aligned}
$$

By using Young's inequality,

$$
\begin{aligned}
& \left(\frac{3}{2}(1+q)-2\right) \int_{M}|B|^{1+2 q} \nabla|B| \cdot \phi \nabla \phi * 1 \\
& \quad \leq \varepsilon \int_{M}|B|^{2 q}|\nabla| B||^{2} \phi^{2} * 1+C_{1}(\varepsilon, q) \int_{M}|B|^{2+2 q}|\nabla \phi|^{2} * 1 .
\end{aligned}
$$

Then (4.4) becomes

$$
\begin{aligned}
\left(\frac{2}{m n}+1+2 q\right. & \left.-\frac{3}{4}(1+q)^{2}-\varepsilon\right) \int_{M}|B|^{2 q}|\nabla| B||^{2} \phi^{2} * 1 \\
& \leq C_{2}(\varepsilon, q) \int_{M}|B|^{2+2 q}|\nabla \phi|^{2} * 1 .
\end{aligned}
$$


When

$$
q \in\left[0, \frac{1}{3}+\frac{2}{3} \sqrt{1+\frac{6}{m n}}\right)
$$

we have

$$
\frac{2}{m n}+1+2 q-\frac{3}{4}(1+q)^{2}>0 .
$$

Then we can choose $\varepsilon$ sufficiently small, such that

$$
\int_{M}|B|^{2 q}|\nabla| B||^{2} \phi^{2} * 1 \leq C_{3} \int_{M}|B|^{2+2 q}|\nabla \phi|^{2} * 1
$$

where $C_{3}$ only depends on $n, m$ and $q$.

Again using Young's inequality yields

$$
|B|^{1+2 q} \nabla|B| \cdot \phi \nabla \phi \leq \frac{1}{2}\left(|B|^{2 q}|\nabla| B||^{2} \phi^{2}+|B|^{2+2 q}|\nabla \phi|^{2}\right) .
$$

Substituting (4.7) and (4.8) into (4.1) gives

$$
\int_{M}|B|^{4+2 q} \phi^{2} * 1 \leq C_{4}(n, m, q) \int_{M}|B|^{2+2 q}|\nabla \phi|^{2} * 1 .
$$

Replacing $\phi$ by $\phi^{2+q}$ in (4.9) gives

$$
\int_{M}|B|^{4+2 q} \phi^{4+2 q} * 1 \leq C_{4}(2+q)^{2} \int_{M}|B|^{2+2 q} \phi^{2+2 q}|\nabla \phi|^{2} * 1 .
$$

By Hölder inequality, we have

$$
\begin{aligned}
& \int_{M}|B|^{2+2 q} \phi^{2+2 q}|\nabla \phi|^{2} * 1 \\
\leq & \left(\int_{M}|B|^{4+2 q} \phi^{4+2 q} * 1\right)^{\frac{1+q}{2+q}}\left(\int_{M}|\nabla \phi|^{4+2 q} * 1\right)^{\frac{1}{2+q}} .
\end{aligned}
$$

Therefore

$$
\int_{M}|B|^{4+2 q} \phi^{4+2 q} * 1 \leq C \int_{M}|\nabla \phi|^{4+2 q} * 1 .
$$

where $C$ is a constant only depending on $n, m$ and $q$.

Replacing $\phi$ by $\phi^{1+q}$ in (4.9) gives

$$
\int_{M}|B|^{4+2 q} \phi^{2+2 q} * 1 \leq C_{4}(1+q)^{2} \int_{M}|B|^{2+2 q} \phi^{2 q}|\nabla \phi|^{2} * 1 .
$$

By Hölder inequality, we have

$$
\begin{aligned}
& \int_{M}|B|^{2+2 q} \phi^{2 q}|\nabla \phi|^{2} * 1 \\
\leq & \left(\int_{M}|B|^{4+2 q} \phi^{2+2 q} * 1\right)^{\frac{q}{1+q}}\left(\int_{M}|B|^{2}|\nabla \phi|^{2+2 q} * 1\right)^{\frac{1}{1+q}} .
\end{aligned}
$$


Therefore

$$
\int_{M}|B|^{4+2 q} \phi^{2+2 q} * 1 \leq C^{\prime} \int_{M}|B|^{2}|\nabla \phi|^{2+2 q} * 1 .
$$

where $C^{\prime}$ is a constant only depending on $n, m$ and $q$.

Let $r$ be a function on $M$ with $|\nabla r| \leq 1$. For any $R \in\left[0, R_{0}\right]$, where $R_{0}=\sup _{M} r$, suppose

is compact.

$$
M_{R}=\{x \in M, \quad r \leq R\}
$$

(4.10) enables us to prove the following results.

Theorem 4.1. Let $M$ be an $n$-dimensional minimal submanifolds of $\mathbb{R}^{n+m}$. If the Gauss image of $M_{R}$ is contained in an open geodesic ball of radius $\frac{\sqrt{2}}{4} \pi$ in $\mathbf{G}_{n, m}$, then we have the $L^{p}$-estimate

$$
\||B|\|_{L^{p}\left(M_{\theta R}\right)} \leq C(n, m, p)(1-\theta)^{-1} R^{-1} \operatorname{Vol}\left(M_{R}\right)^{\frac{1}{p}}
$$

for arbitrary $\theta \in(0,1)$ and

$$
p \in\left[4,4+\frac{2}{3}+\frac{4}{3} \sqrt{1+\frac{6}{m n}}\right) .
$$

Proof. Take $\phi \in C_{c}^{\infty}\left(M_{R}\right)$ to be the standard cut-off function such that $\phi \equiv 1$ in $M_{\theta R}$ and $|\nabla \phi| \leq C(1-\theta)^{-1} R^{-1}$; then (4.10) yields

$$
\int_{M_{\theta R}}|B|^{p} * 1 \leq C(1-\theta)^{-p} R^{-p} \operatorname{Vol}\left(M_{R}\right),
$$

where $p=4+2 q$. Thus the conclusion immediately follows from (4.10).

\section{Curvature estimates of Ecker-Huisken type}

We compute from (2.9) and (3.5):

$$
\begin{aligned}
& \Delta\left(|B|^{2 p} h_{2}^{q}\right) \\
= & \Delta|B|^{2 p} \cdot h_{2}^{q}+|B|^{2 p} \Delta h_{2}^{q}+2 \nabla|B|^{2 p} \cdot \nabla h_{2}^{q} \\
= & \left(p|B|^{2 p-2} \Delta|B|^{2}+\left.\left.p(p-1)|B|^{2 p-4}|\nabla| B\right|^{2}\right|^{2}\right) h_{2}^{q} \\
& +|B|^{2 p}\left(q h_{2}^{q-1} \Delta h_{2}+q(q-1) h_{2}^{q-2}\left|\nabla h_{2}\right|^{2}\right)+4 p q|B|^{2 p-1} \nabla|B| \cdot h_{2}^{q-1} \nabla h_{2} \\
\geq & (4 q-3 p)|B|^{2 p+2} h_{2}^{q}+2 p\left(2 p-1+\frac{2}{m n}\right)|B|^{2 p-2}|\nabla| B||^{2} h_{2}^{q}+q\left(q+\frac{1}{2}\right)|B|^{2 p} h_{2}^{q-2}\left|\nabla h_{2}\right|^{2} \\
& +4 p q|B|^{2 p-1} \nabla|B| \cdot h_{2}^{q-1} \nabla h_{2} .
\end{aligned}
$$

By Young's inequality, when $2 p\left(2 p-1+\frac{2}{m n}\right) \cdot q\left(q+\frac{1}{2}\right) \geq(2 p q)^{2}$, i.e.,

$$
p \geq \frac{1}{2}-\frac{1}{m n}+\left(1-\frac{2}{m n}\right) q,
$$


the inequality

$$
\Delta\left(|B|^{2 p} h_{2}^{q}\right) \geq(4 q-3 p)|B|^{2 p+2} h_{2}^{q}
$$

holds. Especially,

$$
\Delta\left(|B|^{p-1} h_{2}^{\frac{p}{2}}\right) \geq \frac{3}{2}|B|^{p+1} h_{2}^{\frac{p}{2}}
$$

whenever

$$
p \geq m n-1 \text {. }
$$

Let $\eta$ be a smooth function with compact support. Integrating by parts in conjunction with Young's inequality lead to

$$
\begin{aligned}
& \int_{M}|B|^{2 p} h_{2}^{p} \eta^{2 p} * 1 \leq \frac{2}{3} \int_{M}|B|^{p-1} h_{2}^{\frac{p}{2}} \eta^{2 p} \Delta\left(|B|^{p-1} h_{2}^{\frac{p}{2}}\right) * 1 \\
&=-\frac{2}{3} \int_{M} \nabla\left(|B|^{p-1} h_{2}^{\frac{p}{2}} \eta^{2 p}\right) \cdot \nabla\left(|B|^{p-1} h_{2}^{\frac{p}{2}}\right) * 1 \\
&=-\frac{2}{3} \int_{M}\left|\nabla\left(|B|^{p-1} h_{2}^{\frac{p}{2}}\right)\right|^{2} \eta^{2 p} * 1 \\
&(5.5) \quad-\frac{2}{3} \int_{M}|B|^{p-1} h_{2}^{\frac{p}{2}} \cdot 2 p \eta^{2 p-1} \nabla \eta \cdot \nabla\left(|B|^{p-1} h_{2}^{\frac{p}{2}}\right) * 1 \\
& \leq-\frac{2}{3} \int_{M}\left|\nabla\left(|B|^{p-1} h_{2}^{\frac{p}{2}}\right)\right|^{2} \eta^{2 p} * 1+\frac{2}{3} \int_{M}\left|\nabla\left(|B|^{p-1} h_{2}^{\frac{p}{2}}\right)\right|^{2} \eta^{2 p} * 1 \\
& \quad+\frac{2}{3} \int_{M} p^{2}|B|^{2 p-2} h_{2}^{p} \eta^{2 p-2}|\nabla \eta|^{2} * 1 \\
&=\frac{2}{3} p^{2} \int_{M}|B|^{2 p-2} h_{2}^{p} \eta^{2 p-2}|\nabla \eta|^{2} * 1 .
\end{aligned}
$$

By Hölder inequality,

$$
\begin{aligned}
& \int_{M}|B|^{2 p-2} h_{2}^{p} \eta^{2 p-2}|\nabla \eta|^{2} * 1 \\
& =\int_{M}|B|^{2 p-2} h_{2}^{p-1} \eta^{2 p-2} \cdot h_{2}|\nabla \eta|^{2} * 1 \\
& \leq\left(\int_{M}|B|^{2 p} h_{2}^{p} \eta^{2 p} * 1\right)^{\frac{p-1}{p}}\left(\int_{M} h_{2}^{p}|\nabla \eta|^{2 p} * 1\right)^{\frac{1}{p}} .
\end{aligned}
$$

By (5.5) and (5.6), we finally arrive at

$$
\left(\int_{M}|B|^{2 p} h_{2}^{p} \eta^{2 p} * 1\right)^{\frac{1}{p}} \leq \frac{2}{3} p^{2}\left(\int_{M} h_{2}^{p}|\nabla \eta|^{2 p} * 1\right)^{\frac{1}{p}} .
$$

Take $\eta \in C_{c}^{\infty}\left(M_{R}\right)$ to be the standard cut-off function such that $\eta \equiv 1$ in $M_{\theta R}$ and $|\nabla \eta| \leq C(1-\theta)^{-1} R^{-1}$; then from (5.7) we have the following estimate. 
Theorem 5.1. Let $M$ be an $n$-dimensional minimal submanifolds of $\mathbb{R}^{n+m}$. If the Gauss image of $M_{R}$ is contained in an open geodesic ball of radius $\frac{\sqrt{2}}{4} \pi$ in $\mathbf{G}_{n, m}$, then there exists $C_{1}=C_{1}(n, m)$, such that

$$
\left\||B|^{2} h_{2}\right\|_{L^{p}\left(M_{\theta R}\right)} \leq C_{2}(p)(1-\theta)^{-2} R^{-2}\left\|h_{2}\right\|_{L^{p}\left(M_{R}\right)}
$$

whenever $p \geq C_{1}$ and $\theta \in(0,1)$.

Furthermore, the mean value inequality for any subharmonic function on minimal submanifolds in $\mathbb{R}^{m+n}$ (ref. [4], [17]) can be applied to yield an estimate of the upper bound of $|B|^{2}$.

Let $B_{R}(x) \subset \mathbb{R}^{m+n}$ be a ball of radius $R$ and centered at $x \in M$. Its restriction on $M$ is denoted by

$$
D_{R}(x)=B_{R}(x) \cap M
$$

Theorem 5.2. Let $x \in M, R>0$ such that the image of $D_{R}(x)$ under the Gauss map lies in an open geodesic ball of radius $\frac{\sqrt{2}}{4} \pi$ in $\mathbf{G}_{n, m}$. Then, there exists $C_{1}=$ $C_{1}(n, m)$, such that

$$
|B|^{2 p}(x) \leq C(n, p) R^{-(n+2 p)}\left(\sup _{D_{R}(x)} h_{2}\right)^{p} \operatorname{Vol}\left(D_{R}(x)\right),
$$

for arbitrary $p \geq C_{1}$.

Proof. Choose $q=p \geq m n-1$ which satisfies (5.1). The inequality (5.2) means that $|B|^{2 p} h_{2}^{p}$ is a subharmonic function on the minimal submanifold $M$. By Theorem 5.1 and the mean value inequality,

$$
\begin{aligned}
|B|^{2 p} h_{2}^{p}(x) & \leq \frac{C(n)}{\left(\frac{R}{2}\right)^{n}} \int_{D_{\frac{R}{2}}(x)}|B|^{2 p} h_{2}^{p} * 1 \\
& =\frac{C(n)}{\left(\frac{R}{2}\right)^{n}}\left\||B|^{2} h_{2}\right\|_{L^{p}\left(D_{\frac{R}{2}}(x)\right)}^{p} \\
& \leq \frac{C(n) C_{2}(p)^{p}}{\left(\frac{R}{2}\right)^{n+2 p}}\left\|h_{2}\right\|_{L^{p}\left(D_{R}(x)\right)}^{p} \\
& \leq \frac{C(n) C_{2}(p)^{p}}{\left(\frac{R}{2}\right)^{n+2 p}}\left(\sup _{D_{R}(x)} h_{2}\right)^{p} \operatorname{Vol}\left(D_{R}(x)\right)
\end{aligned}
$$

whenever $p \geq C_{1}(n, m)$. 


\section{Geometric conclusions}

Let $P_{0} \in \mathbf{G}_{n, m}$ be a fixed point which is described by

$$
P_{0}=\varepsilon_{1} \wedge \cdots \wedge \varepsilon_{n}
$$

where $\varepsilon_{1}, \cdots, \varepsilon_{n}$ are orthonormal vectors in $\mathbb{R}^{m+n}$. Choose complementary orthonormal vectors $\varepsilon_{n+1}, \cdots, \varepsilon_{n+m}$, such that $\left\{\varepsilon_{1}, \cdots, \varepsilon_{n}, \varepsilon_{n+1}, \cdots, \varepsilon_{n+m}\right\}$ is an orhtonormal base in $\mathbb{R}^{m+n}$.

Let $p: \mathbb{R}^{m+n} \rightarrow \mathbb{R}^{n}$ be the natural projection defined by

$$
p\left(x^{1}, \cdots, x^{n} ; x^{n+1}, \cdots, x^{m+n}\right)=\left(x^{1}, \cdots, x^{n}\right),
$$

which induces a map from $M$ to $\mathbb{R}^{n}$. It is a smooth map from a complete manifold to $\mathbb{R}^{n}$.

For any point $x \in M$ choose a local orthonormal tangent frame field $\left\{e_{1}, \cdots, e_{n}\right\}$ near $x$. Let $v=v_{i} e_{i} \in T M$. Its projection

$$
p_{*} v=\left\langle v_{i} e_{i}, \varepsilon_{j}\right\rangle \varepsilon_{j}=v_{i}\left\langle e_{i}, \varepsilon_{j}\right\rangle \varepsilon_{j} .
$$

For any $P \in \gamma(M)$,

$$
w \stackrel{\text { def. }}{=}\left\langle P, P_{0}\right\rangle=\left\langle e_{1} \wedge \cdots \wedge e_{n}, \varepsilon_{1} \wedge \cdots \wedge \varepsilon_{n}\right\rangle=\operatorname{det} W
$$

where $W=\left(\left\langle e_{i}, \varepsilon_{j}\right\rangle\right)$. It is well known that

$$
W^{T} W=O^{T} \Lambda O
$$

where $O$ is an orthogonal matrix and

$$
\Lambda=\left(\begin{array}{ccc}
\lambda_{1}^{2} & & 0 \\
& \ddots & \\
0 & & \lambda_{r}^{2}
\end{array}\right), \quad r=\min (m, n),
$$

where each $0 \leq \lambda_{i}^{2} \leq 1$.

We now compare the length of any tangent vector $v$ to $M$ with its projection $p_{*} v$.

$$
\left|p_{*} v\right|^{2}=\sum_{j=1}^{n}\left(v_{i}\left\langle e_{i}, \varepsilon_{j}\right\rangle\right)^{2}=(W V)^{T} W V,
$$

where $V=\left(v_{1}, \cdots, v_{n}\right)^{T}$. Hence,

$$
\left|p_{*} v\right|^{2} \geq\left(\lambda^{\prime}\right)^{2}|v|^{2} \geq w^{2}|v|^{2} \geq w_{0}^{2}|v|^{2}
$$

where $\lambda^{\prime}=\min _{i}\left\{\lambda_{i}\right\}$ and $w_{0}=\inf _{M} w$. The induced metric $d s^{2}$ on $M$ from $\mathbb{R}^{m+n}$ is complete, so is the homothetic metric $\tilde{d} s^{2}=w_{0}^{2} d s^{2}$ whenever $w_{0}>0$. (6.1) implies

$$
p:\left(M, \tilde{d} s^{2}\right) \rightarrow\left(\mathbb{R}^{n}, \text { canonical metric }\right)
$$


increases the distance. It follows that $p$ is a covering map from a complete manifold into $\mathbb{R}^{n}$, and a diffeomorphism, since $\mathbb{R}^{n}$ is simply connected. Hence, the induced Riemannian metric on $M$ can be expressed as $\left(\mathbb{R}^{n}, d s^{2}\right)$ with

$$
d s^{2}=g_{i j} d x^{i} d x^{j} .
$$

Furthermore, the immersion $F: M \rightarrow \mathbb{R}^{m+n}$ is realized by a graph $(x, f(x))$ with $f: \mathbb{R}^{n} \rightarrow \mathbb{R}^{m}$ and

$$
g_{i j}=\delta_{i j}+\frac{\partial f^{\alpha}}{\partial x^{i}} \frac{\partial f^{\alpha}}{\partial x^{j}}
$$

At each point in $M$ its image $n$-plane $P$ under the Gauss map is spanned by

$$
f_{i}=\varepsilon_{i}+\frac{\partial f^{\alpha}}{\partial x^{i}} \varepsilon_{\alpha}
$$

It follows that

$$
\left|f_{1} \wedge \cdots \wedge f_{n}\right|^{2}=\operatorname{det}\left(\delta_{i j}+\sum_{\alpha} \frac{\partial f^{\alpha}}{\partial x^{i}} \frac{\partial f^{\alpha}}{\partial x^{j}}\right)
$$

and

$$
\sqrt{g}=\left|f_{1} \wedge \cdots \wedge f_{n}\right|
$$

The $n$-plane $P$ is also spanned by

$$
p_{i}=g^{-\frac{1}{2 n}} f_{i}
$$

furthermore,

$$
\left|p_{1} \wedge \cdots \wedge p_{n}\right|=1
$$

We then have

$$
\begin{aligned}
\left\langle P, P_{0}\right\rangle & =\operatorname{det}\left(\left\langle\varepsilon_{i}, p_{j}\right\rangle\right) \\
& =\left(\begin{array}{ccc}
g^{-\frac{1}{2 n}} & & 0 \\
& \ddots & \\
0 & & g^{-\frac{1}{2 n}}
\end{array}\right) \\
& =\frac{1}{\sqrt{g}} \geq w_{0}
\end{aligned}
$$

and

$$
\sqrt{g} \leq \frac{1}{w_{0}}
$$

Now,

$$
D_{R}(x)=\left\{(\tilde{x}, f(\tilde{x})): \tilde{x} \in \Omega, f_{1}, \cdots, f_{m} \text { are smooth functions on } \Omega\right\}
$$

where $\Omega \subset B_{R} \subset \mathbb{R}^{n}$. Then (6.2) implies

$$
\operatorname{Vol}\left(D_{R}(x)\right) \leq \frac{1}{w_{0}} \cdot \operatorname{Vol}(\Omega) \leq \frac{1}{w_{0}} C(n) R^{n}
$$

The previous arguments show that 
Proposition 6.1. Let $M$ be a complete submanifold in $\mathbb{R}^{m+n}$. If the $w$-function is bounded below by a positive constant $w_{0}$. Then $M$ is an entire graph with Euclidean volume growth. In particular, if the Gauss image of $M$ is contained in a geodesic ball of radius $\frac{\sqrt{2}}{4} \pi$, then $M$ is an entire graph with Euclidean volume growth.

Proof. Now, we consider the case of the image under the Gauss map $\gamma$ containing in an open geodesic ball of radius $\frac{\sqrt{2}}{4} \pi$ and centered at $P_{0}$. The Jordan angles between $P$ and $P_{0}$ are

$$
\theta_{i}=\cos ^{-1}\left(\lambda_{i}\right)
$$

where $\lambda_{i}^{2}$ are eigenvalues of the symmetric matrix $W^{T} W[24]$. We know that

$$
w=\prod \cos \theta_{i}
$$

On the other hand, the distance between $P_{0}$ and $P$ (see [27], pp. 188-194)

$$
d\left(P_{0}, P\right)=\sqrt{\sum \theta_{i}^{2}}
$$

which is less than $\frac{\sqrt{2}}{4} \pi$ by the assumption. It follows that

$$
w>w_{0}=\left(\cos \frac{\sqrt{2}}{4} \pi\right)^{r} .
$$

Theorem 4.1, Schoen-Simon-Yau's type estimates, and Proposition 6.1 give us the following Bernstein type theorem.

Theorem 6.1. Let $M$ be a complete minimal $n$-dimensional submanifold in $\mathbb{R}^{n+m}$ with $n \leq 6$ and $m \geq 2$. If the Gauss image of $M$ is contained in an open geodesic ball of $\mathbf{G}_{n, m}$ centered at $P_{0}$ and of radius $\frac{\sqrt{2}}{4} \pi$, then $M$ has to be an affine linear subspace.

Proof. Now we choose

$$
p=4+\frac{2}{3}+\frac{4}{3} \sqrt{1+\frac{6}{m n}}>6 .
$$

Fix $x \in M$ and let $r$ be the Euclidean distance function from $x$ and $M_{R}=D_{R}(x)$. Hence, letting $R \rightarrow+\infty$ in (4.12) yields

$$
\||B|\|_{L^{p}(M)}=0 .
$$

i.e., $|B|^{2}=0$. $M$ has to be an affine linear subspace.

Theorem 5.2 and Proposition 6.1 yield a Bernstein type result as follows. 
Theorem 6.2. Let $M$ be a complete minimal $n$-dimensional submanifold in $\mathbb{R}^{n+m}$. If the Gauss image of $M$ is contained in an open geodesic ball of $\mathbf{G}_{n, m}$ centered at $P_{0}$ and of radius $\frac{\sqrt{2}}{4} \pi$, and $\left(\frac{\sqrt{2}}{4} \pi-\rho \circ \gamma\right)^{-1}$ has growth

$$
\left(\frac{\sqrt{2}}{4} \pi-\rho \circ \gamma\right)^{-1}=o(R)
$$

where $\rho$ denotes the distance on $\mathbf{G}_{n, m}$ from $P_{0}$ and $R$ is the Euclidean distance from any point in $M$. Then $M$ has to be an affine linear subspace.

Proof. Now we claim

$$
\sec (\sqrt{2} \rho) \leq C\left(\frac{\sqrt{2}}{4} \pi-\rho\right)^{-1}
$$

for a positive constant $C$. It is sufficient to prove the function

$$
t \in\left[0, \frac{\sqrt{2}}{4} \pi\right) \mapsto \sec (\sqrt{2} t)\left(\frac{\sqrt{2}}{4} \pi-t\right)
$$

is bounded, which follows from

$\lim _{t \mapsto\left(\frac{\sqrt{2}}{4} \pi\right)^{-}} \sec (\sqrt{2} t)\left(\frac{\sqrt{2}}{4} \pi-t\right)=\lim _{t \mapsto\left(\frac{\sqrt{2}}{4} \pi\right)^{-}} \frac{\frac{\sqrt{2}}{4} \pi-t}{\cos (\sqrt{2} t)}=\lim _{t \mapsto\left(\frac{\sqrt{2}}{4} \pi\right)^{-}} \frac{-1}{-\sqrt{2} \sin (\sqrt{2} t)}=\frac{\sqrt{2}}{2}$.

Hence we arrive at the inequality

$$
h_{2} \leq C\left(\frac{\sqrt{2}}{4} \pi-\rho \circ \gamma\right)^{-2}
$$

Thus, for any point $x \in M$, by Theorem 5.2 and Proposition 6.1, we have

$$
|B|^{2 p}(x) \leq C(n, p) R^{-2 p}\left(\frac{\sqrt{2}}{4} \pi-\rho \circ \gamma\right)^{-2 p}
$$

Letting $R \rightarrow+\infty$ in the above inequality forces $|B(x)|=0$.

From (4.11) it is easy to obtain the following result.

Theorem 6.3. Let $M$ be an n-dimensional complete minimal submanifolds of $\mathbb{R}^{n+m}$, if the Gauss image of $M$ is contained in an open geodesic ball in $\mathbf{G}_{n, m}$ of radius $\frac{\sqrt{2}}{4} \pi$ and $M$ has finite total curvature, then $M$ has to be an affine linear subspace.

For a minimal $n$-submanifold in $\mathbb{R}^{m+n}$, if its Gauss image is contained in an open geodesic ball on $\mathbf{G}_{n, m}$ of radius $\frac{\sqrt{2}}{4} \pi$, there is a positive function $h_{1}=\cos (\sqrt{2} \rho \circ \gamma)$. Then the strong stability inequality (3.6) follows. Besides its key role in S-S-Y's estimates, there are other applications. We state following results, whose detail proof can be found in the previous paper of the first author [28]. 
Theorem 6.4. Let $M$ be a complete minimal n-submanifold in $\mathbb{R}^{m+n}$. If the image under the Gauss map is contained in an open geodesic ball in $\mathbf{G}_{n, m}$ of radius $\frac{\sqrt{2}}{4} \pi$. Then any $L^{2}$-harmonic 1-form vanishes.

Theorem 6.5. Let $M$ be one as in Theorem 6.4, $N$ be a manifold with non-positive sectional curvature. Then any harmonic map $f: M \rightarrow N$ with finite energy has to be constant.

The argument in [3] and Theorem 6.4 give

Theorem 6.6. Let $M$ be one as in Theorem 6.4. Then $M$ has only one end.

\section{REFERENCES}

1. E. Bombieri, E. De Geogi and E. Guusti: Minimal cones and Bernstein problem. Invent. Math. 7(1969), 243-268.

2. S. Bernstein: Sur un théorème de géométrie et ses application aux équations aux dérivés partielles du type elliptique. Comm. de la Soc Math. de Kharkov (2é sér.) 15 (1915-1917), 38-45.

3. Huai-Dong Cao, Ying Shen and Shunhui Zhu: The structure of stable minimal hypersurfaces in $\mathbb{R}^{n+1}$. Math. Res. Letters 4(1997), 637-644.

4. S. Y. Cheng, P. Li and S. T. Yau: Heat equations on minimal submanifols and their applications. Amer. J. Math. 103(1981), 1021-1063.

5. M. do Carmo and C. K. Peng: Stable complete minimal surfaces in $\mathbb{R}^{3}$ are planes. Bull Amer. Math. Soc.1(1979), 903-906. Acta Math. Sinica, Engl. Ser. 16(2000), 541-548.

6. Qing Chen and Senlin Xu: Rigidity of compact minimal submanifolds in a unit sphere. Geom. Dedicata 45 (1)(1993), 83-88.

7. D. Fischer-Colbrie and R. Schoen: The structure of complete stable minimal surfaces in 3-manifolds of non-negtive scalar curvature. Comm. Pure Appl. Math. 33(1980), 121-132.

8. H. Fujimoto: Modified defect relation for Gauss map of minimal surfaces. J. Differential Geom. 29(1980, 245-262.

9. D. Fischer-Colbrie: Some rigidity theorems for minimal submanifolds of the sphere. Acta math. 145 (1980), 29-46.

10. K. Ecker and G. Huisken: A Bernstein result for minimal graphs of controlled growth. J. Diff. Geom. 31(1990), 397-400.

11. E. Heinz: Über die lösungen der minimalflächengleichung. Nachr. Akad. Wiss. Göttingen Math. Phys. K1 II (1952), 51-56.

12. S. Hildebrandt, J. Jost, J and K. O. Widman: Harmonic mappings and minimal submanifolds Invent.math. 62 (1980), 269-298.

13. J. Jost and Y. L. Xin: Bernstein type theorems for higher codimension Calculus. Var. PDE 9 (1999), 277-296.

14. H. B. Lawson and R. Osserman: Non-existence, non-uniqueness and irregularity of solutions to the minimal surface system. Acta math. 139(1977), 1-17.

15. An-Min Li and Jimin Li: An intrinsic rigidity theorem for minimal submanifolds in a sphere. Arch. Math.58 (1992), 582-594.

16. J. Moser: On Harnack's theorem for elliptic differential equations. Comm. Pure Appl. Math. 14 (1961), 577-591.

17. Lei Ni: Gap theorems for minimal submanifolds in $\mathbb{R}^{n+1}$. Comm. Analy. Geom. 9 (3)(2001), 641-656.

18. R. Osserman: A survey of minimal surfaces. Van Nostrand Reinhold, New York, (1969). 
19. E. A. Ruh and J. Vilms: The tension field of Gauss map. Trans. Amer. Math. 149(1970), 569-573.

20. R. Schoen, L. Simon and S. T. Yau: Curvature estimates for minimal hypersurfaces. Acta Math. 134 (1975), 275-288.

21. J. Simons: Minimal varieties in Riemannian manifolds Ann. Math. 88 (1968), 62-105.

22. K. Smoczyk, Guofang Wang and Y. L. Xin: Bernstein type theorems with flat normal bundle. Calc. Var. and PDE. 26(1)(2006), 57-67.

23. B. Solomon: On the Gauss map of an area-minimizing hypersurface. J. Diff. Geom.19(1984), 221-232.

24. Yung-Chow Wong: Differential geometry of Grassmann manifolds. Proc. N.A.S. 57 (1967), 589-594.

25. F. Xavier: The Gauss map of a complete non-flat minimal surface cannot omit 7 points of the sphere. Ann. Math. 113(1981), 211-214.

26. Y. L. Xin: Geometry of harmonic maps. Birkhäuser PNLDE 23, (1996).

27. Yuanlong Xin: Minimal submanifolds and related topics. World Scientific Publ., (2003).

28. Y. L. Xin: Bernstein type theorems without graphic condition. Asia J. Math. 9(1)(2005), $31-44$.

Institute of Mathematics, Fudan University, Shanghai 200433, China and Key Laboratory of Mathematics for Nonlinear Sciences (Fudan University), Ministry OF EDUCATION

E-mail address: ylxin@fudan.edu.cn 